\title{
Gene Transfer of Heme Oxygenase-1 Using an Adeno-Associated Virus Serotype 6 Vector Prolongs Cardiac Allograft Survival
}

\author{
Jacqueline M. Evans, ${ }^{1}$ Sonia Navarro, ${ }^{1}$ Tomoko Doki, ${ }^{2}$ John M. Stewart, ${ }^{3}$ \\ Noboru Mitsuhashi, ${ }^{2}$ and Mary Kearns-Jonker ${ }^{2,3}$ \\ ${ }^{1}$ Department of Anesthesiology Critical Care Medicine, Children's Hospital Los Angeles, Los Angeles, CA 90027, USA \\ ${ }^{2}$ Department of Cardiothoracic Surgery, Children's Hospital Los Angeles, Los Angeles, CA 90027, USA \\ ${ }^{3}$ Department of Pathology and Human Anatomy, Loma Linda University School of Medicine, Alumni Hall Room 326, \\ 11021 Campus Street, Loma Linda, CA 92350, USA \\ Correspondence should be addressed to Mary Kearns-Jonker, mkearnsjonker@llu.edu
}

Received 18 May 2012; Revised 7 September 2012; Accepted 7 September 2012

Academic Editor: Simon Robson

Copyright (๑) 2012 Jacqueline M. Evans et al. This is an open access article distributed under the Creative Commons Attribution License, which permits unrestricted use, distribution, and reproduction in any medium, provided the original work is properly cited.

Introduction. Allograft survival can be prolonged by overexpression of cytoprotective genes such as heme oxygenase-1 (HO-1). Modifications in vector design and delivery have provided new opportunities to safely and effectively administer HO-1 into the heart prior to transplantation to improve long-term graft outcome. Methods. HO-1 was delivered to the donor heart using an adeno-associated virus vector (AAV) with a pseudotype 6 capsid and vascular endothelial growth factor (VEGF) to enhance myocardial tropism and microvascular permeability. Survival of mouse cardiac allografts, fully or partially mismatched at the MHC, was determined with and without cyclosporine A. Intragraft cytokine gene expression was examined by PCR. Results. The use of AAV6 to deliver HO- 1 to the donor heart, combined with immunosuppression, prolonged allograft survival by $55.3 \%$ when donor and recipient were completely mismatched at the MHC and by $94.6 \%$ if partially mismatched. The combination of gene therapy and immunosuppression was more beneficial than treatment with either AAV6-HO-1 or CsA alone. IL-17a, b, e and f were induced in the heart at rejection. Conclusions. Pretreatment of cardiac allografts with AAV6-HO-1 plus cyclosporine A prolonged graft survival. HO-1 gene therapy represents a beneficial adjunct to immunosuppressive therapy in cardiac transplantation.

\section{Introduction}

Chronic graft vasculopathy (CGV), also referred to as "transplant-associated arteriosclerosis," is a widespread and progressive process characterized by intimal hyperplasia, inflammation, and fibrosis of the graft arterial microvasculature [1]. CGV is a major challenge for heart and other organ transplant recipients [1-4]. Although modern immunosuppressive agents have significantly improved short-term outcomes, long-term outcomes have been less favorable [4]. Ten year survival of adults undergoing heart transplantation is currently at 55\% [5]. Moreover, immunosuppressive therapy is hampered by a multitude of potentially life-threatening side effects as well as the need for lifelong compliance and rigorous monitoring $[4,6]$.
Tissue-directed gene therapy in which a therapeutic molecule is produced within the graft itself could reduce the necessity for systemic immunosuppressive agents [7]. Candidate cytoprotective genes include various heat shock proteins (HSPs) [8], B-cell leukemia/lymphoma-2(bcl-2), nitric oxide synthase, interleukins 4 and $10[9,10]$, and transforming growth factor- $\beta_{1}$ (TGF- $\left.\beta_{1}\right)$ [11]. The well-characterized gene heme oxygenase-1 (HO-1, HSP-32) prevents ischemia reperfusion (I/R) injury [12-17], promotes allograft and xenograft survival $[1,18-24]$, and prevents vascular remodeling [2527]. HO-1 maintains cellular redox status, preserves vascular patency, and inhibits inflammation and apoptosis $[18,28]$.

Adenoviral vector-mediated administration of $\mathrm{HO}-1$ has been shown to prevent chronic rejection of aortic $[29,30]$ and cardiac [31] allografts, but clinical use of adenoviral 
vectors in humans has serious limitations. Wild-type adenovirus is associated with acute respiratory tract infection and administration of adenoviral vectors induces an immune response [32]. In contrast, infection with wild-type adenoassociated virus (AAV) is associated with minimal cellular immune response and is not associated with human pathology [33, 34]. Recombinant AAV (rAAV) vectors have high biosafety profiles and are used in clinical gene therapy trials $[34,35]$. rAAV vectors transduce dividing and nondividing cells and persist as extrachromosomal episomal elements, reducing concerns regarding insertional mutagenesis [33]. Infection with rAAV vectors does not increase mutation rates [36] and is associated with long-lasting gene expression [7, 37-39].

In the heart, earlier generation rAAV pseudotype 2 vectors have been successfully used to administer HO-1 (rAAV2/ HO-1) to prevent I/R injury after myocardial infarction [14, 37-39]. rAAV vectors encoding immunomodulatory or cytoprotective genes may also be beneficial in preventing CGV in patients undergoing heart transplantation [40-44]. The administration of rAAV2 encoding HO-1 modestly prolongs cardiac allograft survival in rats [45]. Later generation vectors, now available, combined with newly identified modifications in vector delivery, may further improve longterm graft outcome. In the study described here, an AAV vector was used to deliver $\mathrm{HO}-1$ to the heart using a later generation pseudotype 6 capsid and coadministration of vascular endothelial growth factor (VEGF) to enhance myocardial tropism and microvascular permeability [46]. Our studies represent the first report comparing graft survival after transplantation of fully and partially mismatched mouse cardiac allografts expressing rAAV6/HO-1. Results from this work may serve as the foundation for future translational studies in which the possibility of HO-1 gene therapy in clinical heart transplantation is explored.

\section{Materials and Methods}

2.1. Construction of Adeno-Associated Viral Vectors. HO-1 cDNA was cloned from mouse splenic lymphocytes. Total RNA was used to prepare cDNA. Primers used to clone the HO-1 gene were 5' AAC TAG CCC AGT CCG GTG ATG GAG C 3' and 5' GGG CCA GTA TTG CAT TTA CAT GGC $3^{\prime}$. We used BLAST to confirm that our cloned gene was mouse HO-1 (NCBI accession number, NM_010442). The HO-1 gene was ligated into the rAAV2 backbone, pAAV2: CMV-MCS-SV40pA (kindly provided by Dr. Michael J. Blankinship, University of Washington, Seattle, WA) and the construct was sequenced. The plasmid pAAV2:CMV-HO-1SV40pA was encapsidated by pA6DG, a packaging/helper plasmid containing a rAAV2 rep and a rAAV6 cap by the vector core at the University of Washington (Seattle, WA). A rAAV6 vector expressing human placental alkaline phosphatase (rAAV6/AP synthesized by Dr. Jeffry Chamberlain, Department of Neurology, University of Washington, Seattle, WA) was used as a control for our studies.

2.2. Western Blot Studies to Confirm Immunoreactivity of Exogenous HO-1. Immunoreactivity of the expressed HO-1 enzyme was confirmed in lysates from transduced minipig kidney (MPK) cells (ATCC, Manassas, VA) and transduced mouse myocardium. MPK cells show no crossreactivity between the antibody for mouse HO-1 and endogenous pig HO-1. To optimize conditions, MPK cells were initially incubated with $2.6 \times 10^{3}$ to $2.6 \times 10^{5}$ vg of rAAV6/ARAP4 and stained for alkaline phosphatase using Sigma SIGMAFAST BCIP/NBT tablets (Sigma-Aldrich, St. Louis, MO). Cells were heated to $65^{\circ} \mathrm{C}$ prior to staining to inactivate endogenous alkaline phosphatase. MPK cells were transduced with $2.6 \times 10^{5} \mathrm{vg}$ of rAAV6/HO-1 for 48 hours and harvested.

For in vivo studies, Balb/cJ mice were injected via tail vein with $2 \times 10^{12}$ vg of rAAV6/HO-1, and hearts were harvested at $14 \mathrm{~d}$ for robust rAAV6-mediated transduction of mouse myocardium [46]. Vector was coadministered with $400 \mathrm{ug} / \mathrm{kg}$ of vascular endothelial growth factor (VEGF, RD Systems, Minneapolis, $\mathrm{MN}$ ) diluted in lactated ringers containing $0.008 \%$ mouse serum albumin (Sigma-Aldrich, St. Louis, $\mathrm{MO})$ and $80 \mathrm{IU} / \mathrm{kg}$ of sodium heparin to increase microvascular permeability [46].

HO-1 immunoreactivity was measured by homogenizing cells and tissue samples in lysis buffer containing $50 \mathrm{mM}$ Tris-HCl, pH 7.4, $20 \mathrm{mM} \mathrm{NaCl}, 10 \mathrm{mM} \mathrm{KCl}, 0.1 \mathrm{mM}$ DTT, $1 \mathrm{mM}$ EDTA pH 8.0 and $0.10 \%$ SDS. Protein concentrations were measured by the Bradford method (Pierce BCA Protein Assay Kit, Pierce Technologies, Rockford, IL). Samples were denatured, separated by sodium dodecyl sulfate polyacrylamide gel electrophoresis (SDS-PAGE), and transferred to polyvinylidene fluoride (PVDF) membranes. Immunoreactive HO- 1 was detected using a rabbit antirat primary antibody $(0.5 \mathrm{ug} / \mathrm{mL}$ or $1: 2000$ dilution $)$ crossreactive for mouse HO-1 (Stressgen, Ann Arbor, MI), a polyclonal HRP-conjugated anti-rabbit secondary antibody ( $0.1 \mathrm{ug} / \mathrm{mL}$ or $1: 10,000$ dilution) (Promega, Madison, WI), and a SuperSignal West Pico Chemiluminescent Kit (Pierce Biotechnology, Rockford, IL). A rabbit polyclonal antibody against actin (Santa Cruz Biotechnologies, Santa Cruz, CA) served as a control.

2.3. Heme Oxygenase Assay to Confirm Functional Activity of Exogenous HO-1. The functional activity of expressed HO1 was verified using a paired enzyme assay for HO-1 activity [47]. HO-1 converts heme to equimolar amounts of biliverdin, carbon monoxide and free iron. Bilverdin is converted to bilirubin by an NADPH-dependent biliverdin reductase. In this assay, an unprocessed liver cytosol preparation $(105,000 \mathrm{~g}$ supernatant from homogenized liver) served as a source of biliverdin reductase. One hundred and forty micrograms of protein from transduced cells or $500 \mathrm{ug}$ of protein from tissue was incubated for 1 hour in the dark at $37^{\circ} \mathrm{C}$. Samples were incubated in $50 \mathrm{uM}$ hemin, $0.8 \mathrm{mM}$ NADPH, $2 \mathrm{mM}$ glucose 6-phosphate (G6P), $1.5 \mathrm{mg}$ of liver cytosol, and $0.2 \mathrm{U}$ G6P dehydrogenase (an NADPH generating system). The reaction was terminated with chloroform. Bilirubin generated through the metabolism of hemin by HO-1 was measured spectrophotometrically at $464 \mathrm{~nm}$ minus the absorbency at $530 \mathrm{~nm}$ (molar extinction coefficient, $\varepsilon=40 \mathrm{mM}^{-1} \mathrm{~cm}^{-1}$ ). HO-1 activity was reported as picomoles of bilirubin generated per mg protein per 
hour. A control reaction containing all components minus the test protein (source of $\mathrm{HO}-1$ ) served as a blank. The spectrophotometric measurements for the blanks were subtracted from all experimental and control samples.

2.4. Distribution Studies. Balb/cJ mice were injected with $2 \times 10^{11} \mathrm{vg}$ of rAAV6/ARAP4, a control vector encoding the reporter gene human placental alkaline phosphatase (AP). Hearts were harvested at 11 days. Five micron frozen sections were stained for AP activity using Sigma SIGMAFAST BCIP/NBT tablets (Sigma-Aldrich, St. Louis, MO).

\subsection{Quantitative PCR to Confirm Persistence of Viral DNA} in Cellular Extracts from Genomic DNA. The persistence of viral DNA in cellular DNA extracts from rAAV6/HO-1transduced hearts was measured at 14 days after treatment of Balb/cJ mice with $2 \times 10^{12}$ or $2 \times 10^{11} \mathrm{vg}$ of $\mathrm{rAAV} 6 / \mathrm{HO}$ 1/animal. Genomic DNA was extracted (Dneasy Tissue Kit, Qiagen, Valencia, CA) and quantified (Sigma QF Kit, SigmaAldrich, St. Louis, MO), against a standard curve. Quantitative PCR (qPCR, Taqman, Applied Biosystems, Pleasanton, CA) was performed using an ABI PRISM 7700 Sequence Detector (Perkin Elmer, Foster City, CA). Primers were designed to target exon junctions to probe for the transgene rather than endogenous DNA. The sequence of the forward and reverse primers and probe were $5^{\prime}$ CAC AGA TGG CGT CAC TTC GT 3', 5' GCG GTG TCT GGG ATG AGC TA 3', and $5^{\prime}$ AGC CTG GTG CAA GAT ACT GCC CC $3^{\prime}$.

2.6. Heterotopic Heart Transplantation. Animal experiments were approved by the Children's Hospital Los Angeles Institutional Animal Care and Use Committee. Mice (Jackson Laboratory, Bar Harbor, ME) were male and 6 to 8 weeks old. Transplantation studies involved fully mismatched cardiac allografts (Balb/cJ, H-2d donors and C57Bl/6J, H-2b recipients, Table 1 ) and partially mismatched cardiac allografts (DBA/2, H-2 $2^{\mathrm{d}}$ donors and B6AF1, H-2 $2^{\mathrm{b} / \mathrm{a}}$ or $\mathrm{H}-2^{\mathrm{b}, \mathrm{k} / \mathrm{d}}$ recipients). Shown in Table $1, \mathrm{DBA} / 2\left(\mathrm{H}-2^{\mathrm{d}}\right)$ mice have a "d" haplotype. Unlike the other strains used in these studies, which are congenic, the B6AF1 strain is an F1 hybrid cross of the B6 ("b" haplotype) and A ("a" haplotype) strains and has a mixed haplotype, $\mathrm{H}-2^{\mathrm{b} / \mathrm{a}}$. The "a" haplotype is a recombinant haplotype and is a cross between the " $k$ " and " $\mathrm{d}$ " haplotypes; therefore, $\mathrm{H}-2^{\mathrm{b} / \mathrm{a}}$ can also be written as $\mathrm{H}-2^{\mathrm{b}, \mathrm{k} / \mathrm{d}}$. The MHC class Ia locus, $\mathrm{K}$ and the MHC class II loci ( $\mathrm{A} \alpha$ and $\beta$ and $\mathrm{E} \alpha$ and $\beta$ ) are " $\mathrm{k}$ " while the MHC class Ia loci, D and $\mathrm{L}$ and the MHC class III locus, $\mathrm{S}$ is "d". Mice were pretreated with $\mathrm{rAAV6/HO}-1$ or the APexpressing control vector rAAV6/ARAP4 or vehicle 9 to 14 days prior to heterotopic heart transplantation. Ventricular contractions were assessed daily by palpation. Rejection was determined by cessation of cardiac function and pathology. Allografts were harvested at rejection (absence of palpable ventricular contractions on abdominal exam) or at 100 days after transplantation, divided for biochemical analyses, snap frozen in liquid nitrogen and stored at $-80^{\circ} \mathrm{C}$.

2.7. Real-Time PCR. Heart tissue was isolated from transplanted animals at the time of rejection or at 100 days after transplantation in animals with long-term surviving grafts. The heart tissue was divided, allowing part of the tissue to be stored in RNA Later (Qiagen, Valencia, CA) and part to be stored snap frozen. RNA was isolated using the RNeasy Fibrous Tissue Kit from Qiagen (Valencia, CA). RNA quality was verified by electrophoresis on $1 \%$ agarose gel and cDNA was then prepared using Superscript III (Invitrogen, Carlsbad, CA). Two ug of RNA was used for each cDNA preparation. Real-time PCR was performed using Fast SYBR Green Master Mix (Promega, Madison, WI) and an IQ 5 Multicolor Real-Time PCR Detection System (BioRad, Hercules, CA). The PCR conditions were $94^{\circ} \mathrm{C}$ for 10 minutes, $94^{\circ} \mathrm{C}$ for 15 seconds, $60^{\circ} \mathrm{C}$ for 60 seconds, and $72^{\circ} \mathrm{C}$ for 30 seconds for a total of 40 cycles. qPCR was run in triplicate. Primers used to identify cytokine expression levels in the heart at the time of rejection or at 100 days in surviving grafts are TNF $\alpha$ FWD AGGAGAAAGTCAACCTCCTCTCTG, REV TGGAAGACTCCTCCCAGGTATATG; IL-2 FWD AAGCATTGCCTTCTAGGTCTCC, REV TCAGAGATACACGAGCTGGTT; IFN- $\gamma$ FWD GCCACGGCACAGTCATTGA, REV TGCTGATGGCCTGATTGTCTT; Il-10 FWDCTTACTGACTGGCATGAGGATCA, REV GCAGCTCTAGGAGCATGTGG, Il-4 FWD GGTCTCAACCCCCAGCTAGT, REV GCCGATGATCTCTCTCAAGTGAT; IL-1 $\beta$ FWDGAAATGCCACCTTTTGACAGTG, REV TGGATGCTCTCATCAGGACAG; IL-17a FWD TCAGCGTGTCCAAACACTGAG, REV CGCCAAGGGAGTTAAAGACTT; Il-17b FWD GAGTAAAGCCCTACGCTCGAA, REVCTCCTCTTGTTGGACAACCAC; IL-17e/IL-25 FWD ACAGGGACTTGAATCGGGTC, REVTGGTAAAGTGGGACGGAGTTG; and IL-17f FWD TGCTACTGTTGATGTTGGGAC,REVCAGAAATGCCCTGGTTTTGGT.

GAPDH FWD GGGCATGGACTGTGGTCATGAG, REV TGCACCACCAACTGCTTAGCC. was used as a housekeeping gene. All primers were well documented with validation information provided at the website http://pga.mgh .harvard.edu/primerbank/. Fold changes in gene expression were determined by comparing the $\mathrm{Ct}$ for each gene (in triplicate). A minimum of three biological replicates were run in each group before and after transplantation. Data was analyzed using the formula $2^{\Delta \Delta \mathrm{Ct}}$ where $\Delta \mathrm{Ct}=\mathrm{Ct}$ of our gene of interest-Ct of GAPDH. The results are shown \pm SEM.

2.8. Statistics. Results were analyzed using ANOVA followed by Bonferroni's Multiple Comparison Test and Kaplan-Meier log-rank analysis (GraphPad PRISM version 5.0, La Jolla, CA). Survival times are listed as mean survival time (MST) \pm SEM. Significant differences in gene expression levels were determined by Student's $t$-test, comparing the $\Delta \Delta \mathrm{CT}$ of the allograft control group versus the fully or semiallogeneic transplant group treated with AAV6-HO-1 + cyclosporine A.

\section{Results}

3.1. Immunoreactivity, Functional Activity, and Distribution of Expressed HO-1. Prior to commencing in vivo studies, immunoreactivity and functional activity of expressed HO-1 was demonstrated in vitro in rAAV6/HO-1-transduced cultured MPK cells. The concentration of rAAV6/HO-1 for 
TABLE 1: MHC H-2 haplotypes of mouse strains used for transplantation.

(a) Complete mismatch

\begin{tabular}{lccccccccc}
\hline & & Class Ia & \multicolumn{2}{c}{ Class II } & \multicolumn{3}{c}{ Class III } & \multicolumn{2}{c}{ Class Ia } \\
Strain & Haplotype & $\mathrm{K}$ & $\mathrm{Ab}$ & $\mathrm{Aa}$ & $\mathrm{Eb}$ & $\mathrm{Ea}$ & $\mathrm{S}$ & $\mathrm{D}$ & $\mathrm{L}$ \\
\hline Balb/cJJ & $\mathrm{d}$ & $\mathrm{d}$ & $\mathrm{d}$ & $\mathrm{d}$ & $\mathrm{d}$ & $\mathrm{d}$ & $\mathrm{d}$ & $\mathrm{d}$ & $\mathrm{d}$ \\
$\mathrm{C} 57 \mathrm{BL} / 6 \mathrm{~J}$ & $\mathrm{~b}$ & $\mathrm{~b}$ & $\mathrm{~b}$ & $\mathrm{~b}$ & $\mathrm{~b}$ & $\mathrm{Null}$ & $\mathrm{b}$ & $\mathrm{b}$ & $\mathrm{Null}$ \\
\hline
\end{tabular}

(b) Partial mismatch

\begin{tabular}{lccccccccc}
\hline & & Class Ia & \multicolumn{3}{c}{ Class II } & \multicolumn{3}{c}{ Class III } & \multicolumn{2}{c}{ Class Ia } \\
Strain & Haplotype & $\mathrm{K}$ & $\mathrm{Ab}$ & $\mathrm{Aa}$ & $\mathrm{Eb}$ & $\mathrm{Ea}$ & $\mathrm{S}$ & $\mathrm{D}$ & $\mathrm{L}$ \\
\hline DBA/2J & $\mathrm{d}$ & $\mathrm{d}$ & $\mathrm{d}$ & $\mathrm{d}$ & $\mathrm{d}$ & $\mathrm{d}$ & $\mathrm{d}$ & $\mathrm{d}$ & $\mathrm{d}$ \\
B6AF1/J & $\mathrm{b} / \mathrm{a}$ & $\mathrm{b} / \mathrm{k}$ & $\mathrm{b} / \mathrm{k}$ & $\mathrm{b} / \mathrm{k}$ & $\mathrm{b} / \mathrm{k}$ & $\mathrm{Null} / \mathrm{k}$ & $\mathrm{b} / \mathrm{d}$ & $\mathrm{b} / \mathrm{d}$ & $\mathrm{b} / \mathrm{d}$ \\
\hline
\end{tabular}

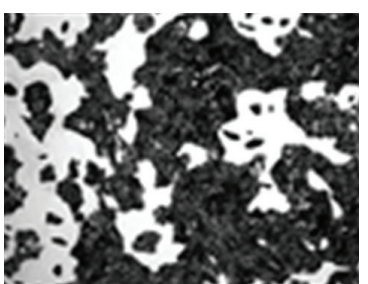

$2.6 \times 10^{5} \mathrm{vg} / \mathrm{cell}$

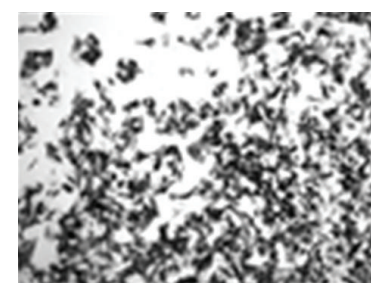

$2.6 \times 10^{4} \mathrm{vg} / \mathrm{cell}$

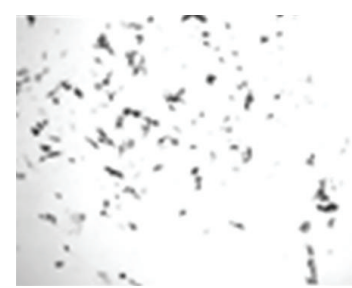

$2.6 \times 10^{3} \mathrm{vg} / \mathrm{cell}$

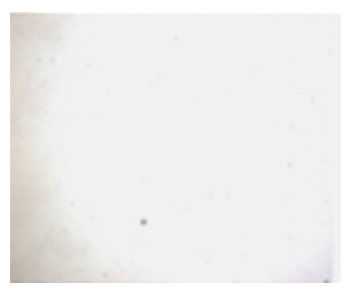

Control

(a)

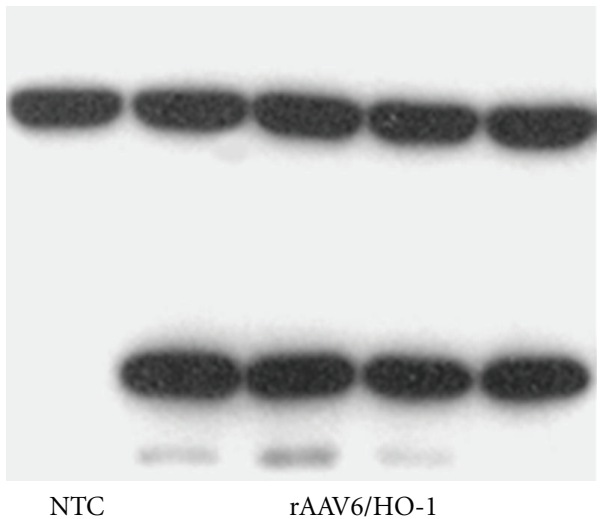

(b)

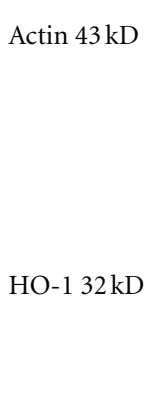

b)

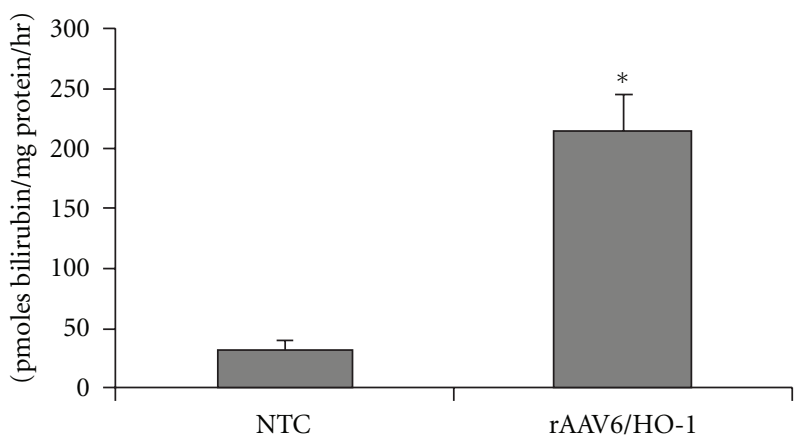

(c)

FIGURE 1: Transduction efficiency in vitro. (a) MPK cells transduced at various concentrations by rAAV6/ARAP4, a control expression vector for the reporter gene alkaline phosphatase (AP), were stained with a histochemical stain for AP 72 hours after transduction. (b) Western blot analysis of HO-1 expression by MPK cells transduced with $2.6 \times 10^{5} \mathrm{VG} /$ cell of rAAV6/HO- 1 for 48 hrs (NTC, nontransduced cells). (c) Enzymatic activity of HO- 1 in MPK cells transduced with $2.6 \times 10^{5} \mathrm{vg} /$ cell of rAAV6/HO- 1 . Values are mean \pm SEM. $N=3$ plates NTC cells, $N=4$ plates rAAV6/HO-1-transduced cells. Each plate was run in triplicate ( ${ }^{*} P<0.003$ as compared to nontransduced control cells, NTC).

in vitro experiments was based on preliminary experiments in which MPK cells were transduced with various concentrations of rAAV6/ARAP4, a control vector encoding the reporter gene human placental alkaline phosphatase (AP) and stained for AP. As shown in Figure 1(a), detectable AP staining was noted after 48-hour incubation with $2.6 \times$ $10^{3}$ viral genomes (vg) of rAAV6/ARAP4 per cell, while $2.6 \times 10^{5} \mathrm{vg} /$ cell showed robust transduction. Incubation of MPK cells with $2.6 \times 10^{5} \mathrm{vg} /$ cell using AAV6/HO- 1 was associated with production of immunoreactive $\mathrm{HO}-1$ by western blot (Figure 1(b)). There was no detectable HO-1 in nontransduced cells. Western blot findings were supported by measurement of HO-1 functional activity. As shown in Figure $1(\mathrm{c})$, incubation of MPK cells with $2.6 \times 10^{5} \mathrm{vg} /$ cell of $\mathrm{rAAV} 6 / \mathrm{HO}-1$ was associated with a 7 -fold increase in functional HO-1 activity versus nontransduced controls.

Expression of HO-1 in myocardium from Balb/cJ mice treated with $2 \times 10^{12} \mathrm{vg}$ of $\mathrm{rAAV} 6 / \mathrm{HO}-1$ was markedly increased at 14 days as compared to expression of HO-1 in nontransduced controls (Figures 2(a) and 2(b)). Functional HO-1 activity [47] was 10-fold greater than that observed in hearts from nontransduced controls (Figure 2(c)). Separate 


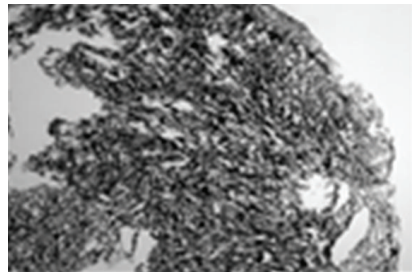

rAAV6/ARAP4

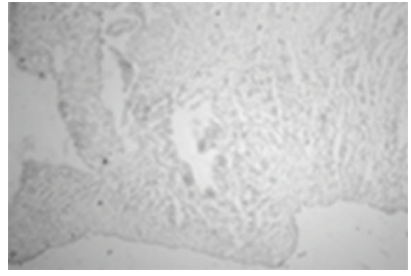

Control

(a)

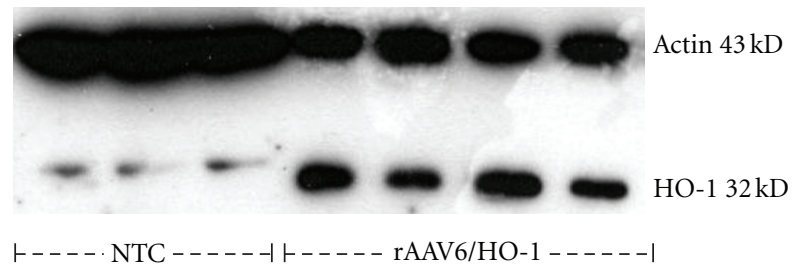

(b)

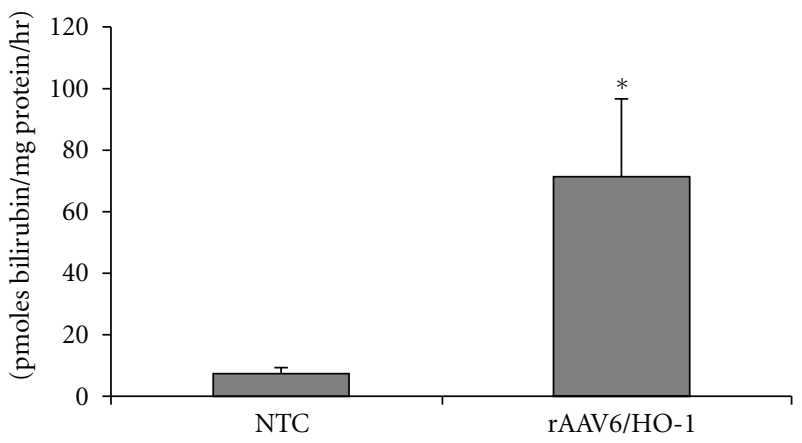

(c)

FIGURE 2: Gene transfer in vivo. (a) Alkaline phosphatase staining of Balb/cJ hearts after intravenous injection of $2 \times 10^{11}$ viral genome (VG) particles of rAAV6/ARAP4 as compared to untreated controls. (b) Western blot of hearts from Balb/cJ mice treated with $2 \times 10^{12} \mathrm{vg}$ of rAAV6/HO-1 as compared to nontransduced controls (NTC). (c) HO-1 functional activity in hearts from Balb/cJ mice treated with $2 \times 10^{12}$ vg of rAAV6/HO-1 as compared to nontransduced controls (NTC). NTC, $N=3$, rAAV6/HO- $1, N=4$. Each sample was run in triplicate (NTC) or quadruplicate (rAAV6/HO-1, ${ }^{*} P<0.05$ as compared to nontransduced control cells, NTC).

experiments were performed to measure the myocardial distribution of vector. Hearts from rAAV6/ARAP4-treated animals showed widespread and homogenous staining for AP activity. There was no detectable staining in hearts from nontransduced control animals.

\subsection{Persistence of Viral DNA in Cellular Extracts from Genom-} ic DNA. The persistence of viral DNA in extracts of genomic DNA from hearts of Balb/cJ mice transduced with either $2 \times 10^{11}$ or $2 \times 10^{12} \mathrm{vg}$ of $\mathrm{rAAV} 6 / \mathrm{HO}-1$ was measured at 14 days. As shown in Figure 3, systemic administration of rAAV6/HO-1 increased levels of viral DNA in the heart identified by Q-PCR in a dose responsive manner. Levels of viral DNA were nondetectable in nontransduced control animals and were elevated in extracts from the hearts of animals receiving $2 \times 10^{12} \mathrm{vg}$ as compared to those receiving $2 \times 10^{11} \mathrm{vg}$ of rAAV6/HO-1.

3.3. The Effect of $r A A V 6 / H O-1$ on Allograft Survival. The effect of transduction of the donor heart with rAAV6/HO1 on allograft survival was assessed after transplantation in donor/recipient combinations that were completely or partially mismatched at the MHC loci (Table 1, Figure 4) [48]. Transduced hearts from Balb/cJ mice were transplanted into C57Bl/6J recipients, and graft survival was compared with syngeneic cardiac graft controls. All grafts transplanted into syngeneic controls $(\mathrm{C} 57 \mathrm{Bl} / 6 \mathrm{~J}$ hearts into $\mathrm{C} 57 \mathrm{Bl6} / \mathrm{J}$ recipients, $n=4$ ) survived for the entire 100-day study period (data not shown) whereas the mean survival time (MST) for grafts from nontransduced control Balb/cJ mice transplanted into $\mathrm{C} 57 \mathrm{Bl} / 6 \mathrm{~J}$ recipients was $7.4 \pm 0.4$ days $(n=9)$ (Figure 4$)$. Intravenous (i.v.) administration of $2 \times 10^{11} \mathrm{vg}$ of rAAV6/ HO-1 eleven days prior to transplantation did not significantly prolong graft survival (MST $8.5 \pm 0.6$ days, $n=4$ ). Treatment of recipients with the immunosuppressant drug CsA to block T-cell activation has been shown to prolong HO-1-mediated cardiac allograft survival in xenograft and allograft models $[45,49]$. In subsequent experiments, systemic administration of $2 \times 10^{12} \mathrm{vg}$ of $\mathrm{rAAV} 6 / \mathrm{HO}-1$ to donor mice 14 days prior to transplantation was combined with intraperitoneal (i.p.) injection of recipients with $15 \mathrm{mg} / \mathrm{kg}$ of CsA on days 0-10. Treatment of recipients with CsA prior to transplantation of $\mathrm{rAAV} 6 / \mathrm{HO}-1$-transduced cardiac 


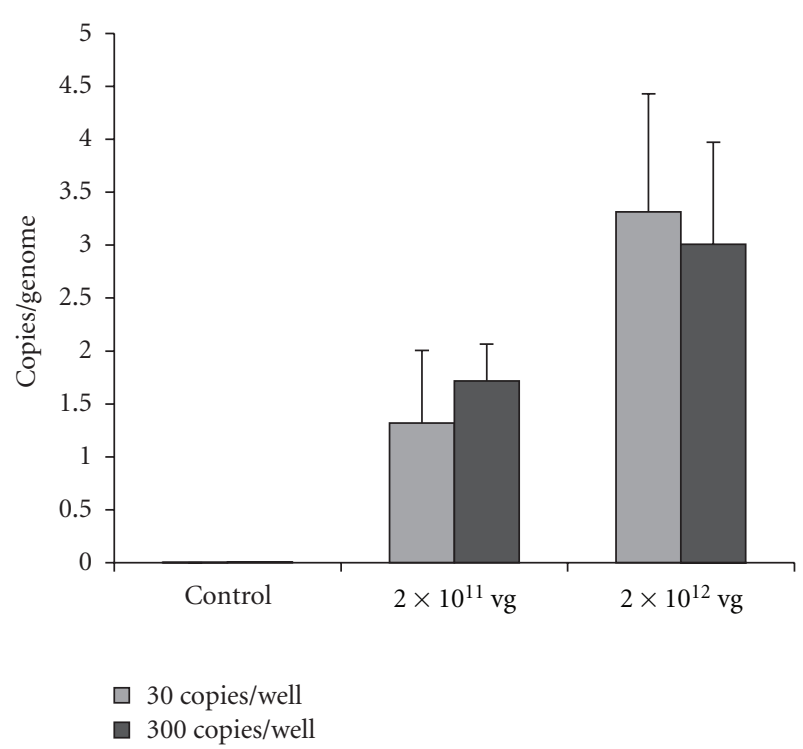

Figure 3: Quantitation of viral genomes in hearts. Heart transduction efficiency ratio was determined based on the amount of vector genome copy numbers in the hearts transduced with $2 \times 10^{11}$ $(n=3)$ or $2 \times 10^{12}(n=6) \mathrm{vg}$ of rAAV6/HO- 1 as compared to nontransduced control animals. Samples were run at 30 genome copies per well (grey bars) and 300 genome copies per well (black bars). Each animal sample was done in triplicates. Results are mean \pm SEM for each sample.

allografts significantly prolonged MST $(13.2 \pm 1.8$ days, $55.3 \%$ increase, $n=5$ ) compared to survival of nontransduced control hearts in untreated recipients (Figure 4(a)). Treatment with CsA prior to transplantation of hearts without genetic modification demonstrated a MST of 9.8 days \pm 1.6 days $(n=8)$.

In order to assess whether $\mathrm{AAV} / \mathrm{HO}-1$ transduction of the donors combined with CsA-mediated immunosuppression of the recipients would have a greater effect when the MHCs of the donor and recipient were only partially mismatched, hearts from DBA/2 donors were transplanted into B6AF1 recipients (Figure 4(b)). All grafts transplanted into syngeneic control animals (B6AF1 hearts into B6AF1 recipients, $n=4$ ) survived for the entire 100-day study period (data not shown) whereas the MST for grafts from nontransduced control DBA/2 mice transplanted into B6AF1 recipients was $24.1 \pm 12.9$ days $(n=7)$. Transduction of donors with $1 \times 10^{12} \mathrm{vg}$ of rAAV6/HO-1 nine days prior to transplantation did not prolong graft survival (MST $10.3 \pm 0.3$ days, $n=3$ ). The combination of i.v. administration of $1 \times 10^{12} \mathrm{vg}$ of rAAV6/HO-1 to donor mice 9 days prior to transplantation and treatment of recipients with $15 \mathrm{mg} / \mathrm{kg}$ (i.p.) of CsA on days $0-10$, however, significantly augmented graft survival to a MST of $46.8 \pm 13.8$ days, a $94.6 \%$ increase in MST compared with controls $(n=9)$. Treatment with CsA alone prior to transplantation of non-transduced allografts did not significantly prolong cardiac graft survival (MST $29.7+9.6$, $n=9)$.

3.4. Intragraft Cytokine Gene Expression. The local cytokine milieu has a role in inducing naive $\mathrm{CD} 4+\mathrm{T}$ helper cells to differentiate towards T helper 1 (Th1), Th2, Th17 and regulatory $\mathrm{T}$ cells. Skewing the immune response towards Th17 or Th1 and away from $\mathrm{T}$ regulatory responses is associated with graft rejection whereas high levels of IL-4 and IL-10 are beneficial to graft survival. Real-time PCR was used to examine cytokine gene expression changes after transplantation of hearts in each of five groups, including (1) hearts that were not genetically modified and represented a donor/host combination that was fully mismatched at the MHC, (2) hearts that were not genetically modified and were partially mismatched at the MHC when compared to their recipients, (3) genetically modified AAV6-HO-1 overexpressing hearts that were fully mismatched at the MHC after transplantation into mice treated with cyclosporine A, (4) genetically modified AAV6-HO-1 hearts that were transplanted in a partial mismatch combination in recipients treated with cyclosporine A and (5) untreated normal hearts. Fold changes in gene expression were determined relative to transplanted hearts in the absence of genetic modification of the donor heart and were also calculated relative to control, untreated hearts.

Interferon- $\gamma$ and IL- $1 \beta$ transcripts were induced at rejection in hearts without genetic modification. Hearts overexpressing HO-1 demonstrated a shift in cytokine expression within the graft. IL-4 and IL-10 transcripts were significantly elevated. IL-10 is a potent anti-inflammatory cytokine whose expression antagonizes Th1 responses via inhibition of antigen presentation and inhibition of interferon gamma production [50]. The cytokines interferon- $\gamma$ and IL- $1 \beta$ were significantly downregulated in the AAV6-HO-1 group.

Intragraft cytokine levels in mice rejecting fully allogeneic grafts that were treated with $\mathrm{HO}-1$ could be distinguished from mice accepting their grafts in the semiallogeneic model by an $8-22$-fold elevation $(P<0.05)$ in IL-17 transcript levels at the time of rejection (Figure 5).

\section{Discussion}

HO-1 plays an important role in mitigating I/R injury, preventing transplant arteriosclerosis and prolonging cardiac allograft and xenograft survival $[18,21,24,49,51,52]$. Effective and safe methods of delivery that can be translated into clinical applications will require careful studies to identify vectors with durable expression and a tolerable risk profile [7]. Routes of administration used to deliver genes to donor hearts include intracoronary infusion, intramyocardial and endomyocardial injection, pericardial instillation and systemic administration [31, 46, 53, 54]. Recently, Gregorevic and colleagues demonstrated that robust and widespread myocardial gene expression could be achieved after a single systemic injection of the donor with a rAAV6 vector administered with VEGF to increase vascular permeability [46]. We were interested in determining whether HO1 gene transfer using a rAAV6 construct and this route of administration would improve long-term cardiac allograft survival.

Recombinant adenoviral vectors have been used in numerous gene therapy studies for heart transplantation, but they do not integrate into the host genome and are associated 


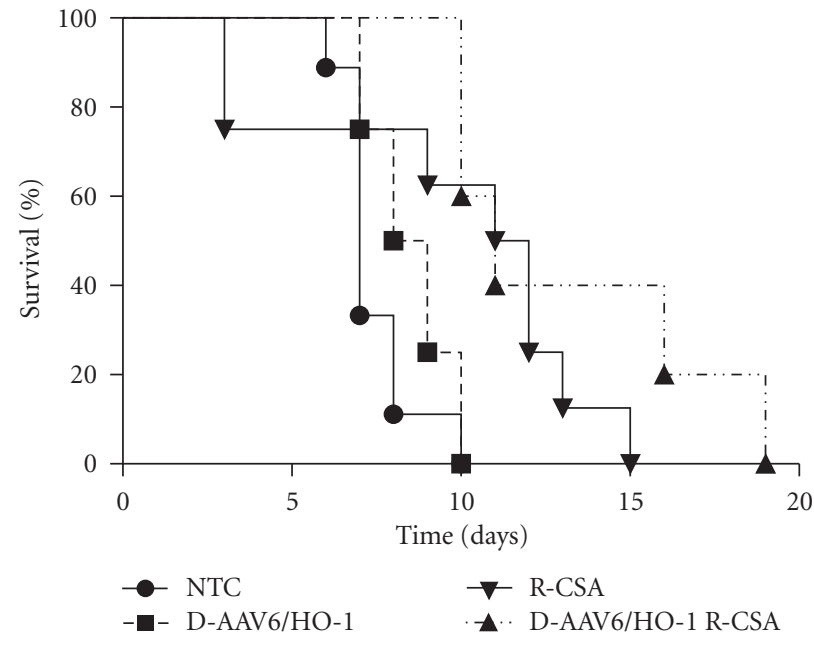

(a)

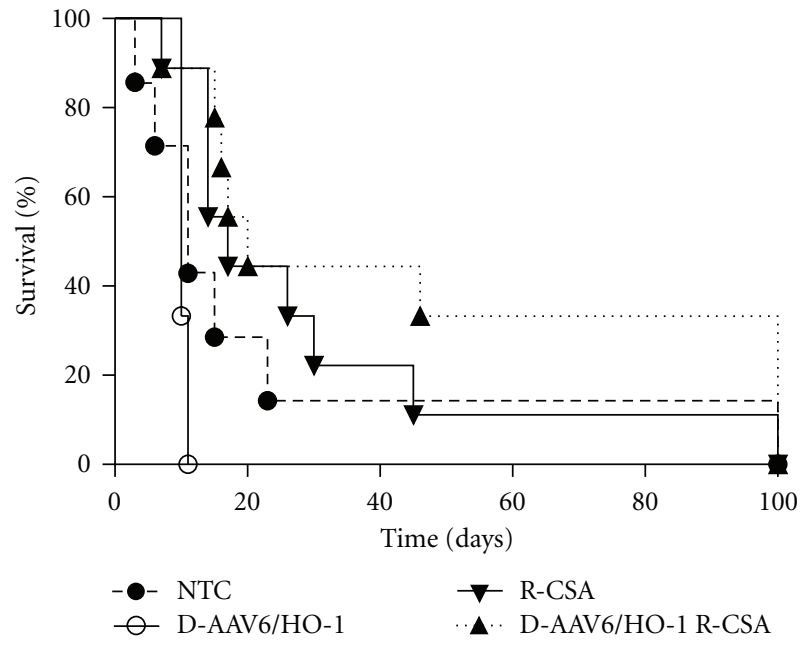

(b)

FIGURE 4: Allograft survival. (a) Graft survival in various treatment groups after transplantation with fully allogeneic hearts. The recipients (R) were male C57BL/6J mice and the donors (D) were male Balb/cJ hearts. Long term graft survival was significantly greater after AAV/HO1 plus CSA treatment $(\boldsymbol{\Lambda}, n=5, P=0.0421)$ compared with AAV/HO-1 only $(\boldsymbol{\square}, n=4)$, CSA treatment only $(\boldsymbol{\nabla}, n=8)$, and controls $(\bullet, n=10)$. (b) In the semiallogeneic model, recipient male B6AF1/J mice were transplanted with donor male DBA/2J hearts. The effect of AAV/HO-1 pre-treatment of the donor and CSA treatment of the recipient was longer-term cardiac allograft survival $(\boldsymbol{\Lambda}, n=9, P=0.064)$ compared with graft survival in the AAV/HO-1-treated group $(\circ, n=3)$ or the CSA-treated group without HO-1 $(\boldsymbol{\nabla}, n=9)$. Nontransduced controls are shown as $(\bullet, n=7)$.

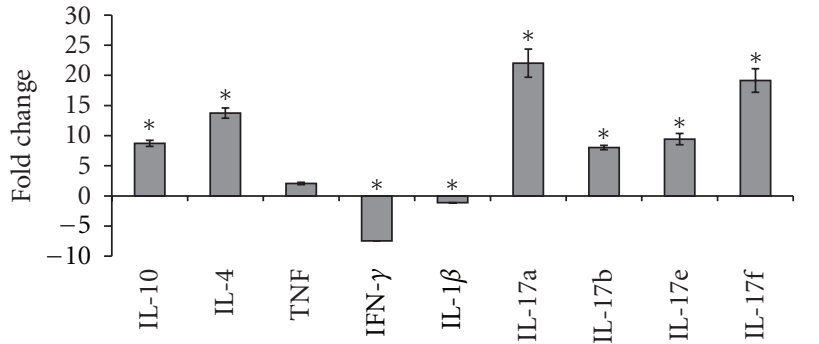

(a)

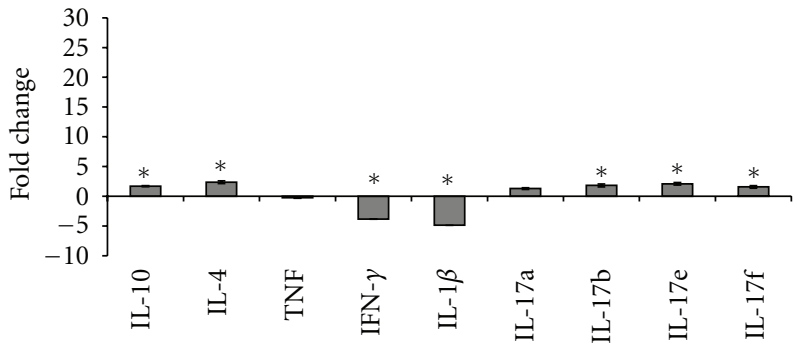

(b)

FIGURE 5: Intragraft cytokine gene expression. Real-time PCR was used to examine cytokine gene expression changes after transplantation of hearts that were (a) fully mismatched at the MHC or (b) partially mismatched at the MHC when compared to their recipients. Interferon- $\gamma$ was not significantly expressed in the transduced hearts but was highly expressed in hearts that were not genetically modified prior to transplantation. IL-17a, IL-17b, IL-17e, and IL-17f were upregulated at rejection $\left({ }^{*} P<0.05\right.$ comparing $\Delta \Delta \mathrm{CT}$ allograft versus HO-1 allograft + cyclosporine (a). IL-4 and IL-10 transcripts were significantly elevated after AAV6-HO-1 transduction of the donor hearts, IL-1 $\beta$ was significantly downregulated when compared with nontransduced control allografts. The upregulated genes $=\Delta \Delta \mathrm{CT}($ Experimental $) / \Delta \Delta \mathrm{CT}(\mathrm{Control})$ where $\Delta \Delta \mathrm{CT}=2^{-(\mathrm{GOI}-\mathrm{HKG})}$. The downregulated genes $=\log _{2}(\Delta \Delta \mathrm{CT}($ Experimental $) / \Delta \Delta \mathrm{CT}(\mathrm{Control}))$ where $\Delta \Delta \mathrm{CT}=2^{-(\mathrm{GOI}-\mathrm{HKG})}$. Samples were run in triplicate and represent a minimum of three biological replicates from each group.

with transient gene expression ( $\sim 4$ weeks). Immune responses hamper the efficacy of gene therapy using adenoviral vectors due to elimination of cells expressing viral antigens by cytotoxic $\mathrm{T}$ cells and an inability to readminister vector due to the presence of neutralizing antibody arising from previous infections of wild-type adenovirus $[7,32,55]$.

In contrast, infection with wild-type AAV is safe, effective and induces a minimal cellular immune response [33-35, 56]. These vectors are undergoing continual improvement. Later generation AAV vectors have been reported to be highly efficient at transducing rodent skeletal muscle and myocardium $[46,56,57]$. In the current study, a single systemic dose of a rAAV6 vector encoding $\mathrm{HO}-1$ was used to transduce donor hearts in vivo. A rAAV vector with a pseudotype 6 capsid was chosen because of its clinical applicability and strong cardiac tropism as compared to pseudotype 2 vectors [55]. Zincarelli et al. recently compared adenoassociated viral vector serotypes 1-9 and reported that AAV6 was most efficient for high level transduction of the mouse myocardium in a tissue-specific manner [56]. Our report is the first use of a rAAV6 vector overexpressing HO-1 in a cardiac model in which survival of fully or partially mismatched allografts is compared. Braudeau and coworkers observed enhanced survival of cardiac allografts after 
intragraft (13.3\%), intramuscular (62.5\%), and i.v. (80\%) administration of an adenoviral vector encoding HO-1 in rats completely mismatched at the MHC loci [31]. Tsui and coworkers used an AAV2 vector to transduce cardiac allografts in rats partially mismatched at the MHC [45] and achieved $60 \%$ survival of transduced grafts. Survival was increased to $89 \%$ by additional treatment with CsA [45]. In our study, rAAV6-HO-1 pretreatment of the donor heart, combined with immunosuppression of the recipient, prolonged allograft survival by $55.3 \%$ when donor and recipient were completely mismatched at the MHC and by $94.6 \%$ when donor and recipient were partially mismatched at the MHC.

Prolonged graft survival was associated with intragraft changes in cytokine expression. We and others have shown that elevated levels of IL4 and IL10 can be identified in longterm surviving grafts, but the contribution of IL-17 subsets to graft survival/rejection after cytoprotective gene transfer has not, to our knowledge, been reported previously. IL17 has recently been identified as a major contributor to graft rejection as demonstrated in several models, including knockout mice (reviewed in [58-60]). There are six IL-17 family members (IL-17 A-F). In our model, IL-17a, b, e and $f$ were notably elevated at the time of graft rejection. IL-17a and IL-17f have been reported to induce proinflammatory cytokines and neutrophil infiltration [61]. The proinflammatory response was similarly elevated at rejection in our study. Transcripts encoding all subsets of IL-17 remained unchanged relative to the native heart in long-term surviving grafts overexpressing $\mathrm{HO}-1$.

Our data support the conclusion that pre-treatment of cardiac allografts with AAV6-HO-1 provides an added benefit and significantly prolongs graft survival in cyclosporine A-treated recipients. The inability to significantly prolong graft survival via HO-1 overexpression alone in the current study may be due to the greater stringency of the mouse as compared to the rat MHC system.

\section{Abbreviations}

rAAV6 vector: Recombinant adenovirus-associated serotype 6 vector

HO-1: Heme oxygenase-1.

\section{Acknowledgment}

This work Supported by a Research Career Development Award from The Saban Research Institute, Children's Hospital Los Angeles to J. M. Evans.

\section{References}

[1] M. Velez and M. R. Johnson, "Management of allosensitized cardiac transplant candidates," Transplantation Reviews, vol. 23, no. 4, pp. 235-247, 2009.

[2] I. Kaczmarek, M. A. Deutsch, T. Kauke et al., "Donor-specific HLA alloantibodies: long-term impact on cardiac allograft vasculopathy and mortality after heart transplant," Experimental and Clinical Transplantation, vol. 6, no. 3, pp. 229-235, 2008.
[3] C. Larosa, B. H. Jorge, and K. E. C. Meyers, "Outcomes in pediatric solid-organ transplantation," Pediatric Transplantation, vol. 15, no. 2, pp. 128-141, 2011.

[4] M. N. Scherer, B. Banas, K. Mantouvalou et al., "Current concepts and perspectives of immunosuppression in organ transplantation," Langenbeck's Archives of Surgery, vol. 392, no. 5, pp. 511-523, 2007.

[5] J. D. Vega, J. Moore, S. Murray, J. M. Chen, M. R. Johnson, and D. B. Dyke, "Heart transplantation in the United States, 1998-2007," American Journal of Transplantation, vol. 9, no. 4, pp. 932-941, 2009.

[6] H. Schrem, H. Barg-Hock, C. P. Strassburg, A. Schwarz, and J. Klempnauer, "Aftercare for patients with transplanted organs," Deutsches Arzteblatt, vol. 106, no. 9, pp. 148-156, 2009.

[7] G. Vassalli, S. Fleury, J. Li, J. J. Goy, L. Kappenberger, and L. K. Von Segesser, "Gene transfer of cytoprotective and immunomodulatory molecules for prevention of cardiac allograft rejection," European Journal of Cardio-thoracic Surgery, vol. 24, no. 5, pp. 794-806, 2003.

[8] B. K. Brar, A. Stephanou, M. J. D. Wagstaff et al., "Heat shock proteins delivered with a virus vector can protect cardiac cells against apoptosis as well as against thermal or hypoxic stress," Journal of Molecular and Cellular Cardiology, vol. 31, no. 1, pp. 135-146, 1999.

[9] S. Fu, D. Chen, X. Mao, N. Zhang, Y. Ding, and J. S. Bromberg, "Feline immunodeficiency virus-mediated viral interleukin10 gene transfer prolongs non-vascularized cardiac allograft survival," American Journal of Transplantation, vol. 3, no. 5, pp. 552-561, 2003.

[10] H. Furukawa, K. Oshima, T. Tung, G. Cui, H. Laks, and L. Sen, "Liposome-mediated combinatorial cytokine gene therapy induces localized synergistic immunosuppression and promotes long-term survival of cardiac allografts," Journal of Immunology, vol. 174, no. 11, pp. 6983-6992, 2005.

[11] R. Brauner, M. Nonoyama, H. Laks et al., "Intracoronary adenovirus-mediated transfer of immunosuppressive cytokine genes prolongs allograft survival," Journal of Thoracic and Cardiovascular Surgery, vol. 114, no. 6, pp. 923-933, 1997.

[12] M. Katori, R. Buelow, B. Ke et al., "Heme oxygenase-1 overexpression protects rat hearts from cold ischemia/reperfusion injury via an antiapoptotic pathway," Transplantation, vol. 73, no. 2, pp. 287-292, 2002.

[13] Y. Akamatsu, M. Haga, S. Tyagi et al., "Heme oxygenase1-derived carbon monoxide protects hearts from transplant associated ischemia reperfusion injury," The FASEB Journal, vol. 18, no. 6, pp. 771-772, 2004.

[14] L. G. Melo, R. Agrawal, L. Zhang et al., "Gene therapy strategy for long-term myocardial protection using adeno-associated virus-mediated delivery of heme oxygenase gene," Circulation, vol. 105, no. 5, pp. 602-607, 2002.

[15] S. R. Vulapalli, Z. Chen, B. H. L. Chua, T. Wang, and C. S. Liang, "Cardioselective overexpression of HO-1 prevents I/Rinduced cardiac dysfunction and apoptosis," American Journal of Physiology, vol. 283, no. 2, pp. H688-H694, 2002.

[16] S. F. Yet, R. Tian, M. D. Layne et al., "Cardiac-specific expression of heme oxygenase-1 protects against ischemia and reperfusion injury in transgenic mice," Circulation Research, vol. 89, no. 2, pp. 168-173, 2001.

[17] J. Ma, C. K. Lau, A. Obed et al., "A cell penetrating heme oxygenase protein protects heart graft against ischemia/reperfusion injury," Gene Therapy, vol. 16, no. 3, pp. 320$328,2009$. 
[18] M. Katori, R. W. Busuttil, and J. W. Kupiec-Weglinski, "Heme oxygenase-1 system in organ transplantation," Transplantation, vol. 74, no. 7, pp. 905-912, 2002.

[19] S. G. Tullius, M. Nieminen-Kelhä, R. Buelow et al., "Inhibition of ischemia/reperfusion injury and chronic graft deterioration by a single-donor treatment with cobalt-protoporphyrin for the induction of heme oxygenase-1," Transplantation, vol. 74, no. 5, pp. 591-598, 2002.

[20] M. P. Soares, Y. Lin, J. Anrather et al., "Expression of heme oxygenase-1 can determine cardiac xenograft survival," Nature Medicine, vol. 4, no. 9, pp. 1073-1077, 1998.

[21] W. W. Hancock, R. Buelow, M. H. Sayegh, and L. A. Turka, "Antibody-induced transplant arteriosclerosis is prevented by graft expression of anti-oxidant and anti-apoptotic genes," Nature Medicine, vol. 4, no. 12, pp. 1392-1396, 1998.

[22] K. Yamashita, R. Öllinger, J. McDaid et al., "Heme oxygenase-1 is essential for and promotes tolerance to transplanted organs," FASEB Journal, vol. 20, no. 6, pp. 776-778, 2006.

[23] J. A. Araujo, L. Meng, A. D. Tward et al., "Systemic rather than local heme oxygenase-1 overexpression improves cardiac allograft outcomes in a new transgenic mouse," Journal of Immunology, vol. 171, no. 3, pp. 1572-1580, 2003.

[24] L. A. Debruyne, J. C. Magee, R. Buelow, and J. S. Bromberg, "Gene transfer of immunomodulatory peptides correlates with heme oxygenase-1 induction and enhanced allograft survival," Transplantation, vol. 69, no. 1, pp. 120-128, 2000.

[25] D. A. Tulis, W. Durante, K. J. Peyton, A. J. Evans, and A. I. Schafer, "Heme oxygenase-1 attenuates vascular remodeling following balloon injury in rat carotid arteries," Atherosclerosis, vol. 155, no. 1, pp. 113-122, 2001.

[26] H. J. Duckers, M. Boehm, A. L. True et al., "Heme oxygenase1 protects against vascular constriction and proliferation," Nature Medicine, vol. 7, no. 6, pp. 693-698, 2001.

[27] L. E. Otterbein, B. S. Zuckerbraun, M. Haga et al., "Carbon monoxide suppresses arteriosclerotic lesions associated with chronic graft rejection and with balloon injury," Nature Medicine, vol. 9, no. 2, pp. 183-190, 2003.

[28] W. Durante, "Heme oxygenase-1 in growth control and its clinical application to vascular disease," Journal of Cellular Physiology, vol. 195, no. 3, pp. 373-382, 2003.

[29] C. Chauveau, D. Bouchet, J. C. Roussel et al., "Gene transfer of heme oxygenase-1 and carbon monoxide delivery inhibit chronic rejection," American Journal of Transplantation, vol. 2, no. 7, pp. 581-592, 2002.

[30] D. Bouchet, C. Chauveau, J. C. Roussel et al., "Inhibition of graft arteriosclerosis development in rat aortas following heme oxygenase-1 gene transfer," Transplant Immunology, vol. 9, no. 2-4, pp. 235-238, 2001.

[31] C. Braudeau, D. Bouchet, L. Tesson et al., "Induction of longterm cardiac allograft survival by heme oxygenase- 1 gene transfer," Gene Therapy, vol. 11, no. 8, pp. 701-710, 2004.

[32] Y. Yang, Q. Li, H. C. J. Ertl, and J. M. Wilson, "Cellular and humoral immune responses to viral antigens create barriers to lung-directed gene therapy with recombinant adenoviruses," Journal of Virology, vol. 69, no. 4, pp. 2004-2015, 1995.

[33] P. E. Monahan and R. J. Samulski, "Adeno-associated virus vectors for gene therapy: more pros than cons?" Molecular Medicine Today, vol. 6, no. 11, pp. 433-440, 2000.

[34] C. Mueller and T. R. Flotte, "Clinical gene therapy using recombinant adeno-associated virus vectors," Gene Therapy, vol. 15 , no. 11, pp. 858-863, 2008.

[35] S. Daya and K. I. Berns, "Gene therapy using adeno-associated virus vectors," Clinical Microbiology Reviews, vol. 21, no. 4, pp. 583-593, 2008.
[36] D. G. Miller, L. M. Petek, and D. W. Russell, "Adeno-associated virus vectors integrate at chromosome breakage sites," Nature Genetics, vol. 36, no. 7, pp. 767-773, 2004.

[37] Q. Li, Y. Guo, Q. Ou et al., "Gene transfer as a strategy to achieve permanent cardioprotection II: rAAV-mediated gene therapy with heme oxygenase-1 limits infarct size 1 year later without adverse functional consequences," Basic Research in Cardiology, vol. 106, no. 6, pp. 1367-1377, 2011.

[38] A. S. Pachori, L. G. Melo, L. Zhang, S. D. Solomon, and V. J. Dzau, "Chronic recurrent myocardial ischemic injury is significantly attenuated by pre-emptive adeno-associated virus heme oxygenase-1 gene delivery," Journal of the American College of Cardiology, vol. 47, no. 3, pp. 635-643, 2006.

[39] X. Liu, A. S. Pachori, C. A. Ward et al., "Heme oxygenase1 (HO-1) inhibits postmyocardial infarct remodeling and restores ventricular function," FASEB Journal, vol. 20, no. 2, pp. 207-216, 2006.

[40] L. Burdorf, N. Schuhmann, J. Postrach et al., "AAV-mediated gene transfer to cardiac cells in a heterotopic rat heart transplantation model," Transplantation Proceedings, vol. 39, no. 2, pp. 567-568, 2007.

[41] B. Asfour, H. A. Baba, H. H. Scheld, R. H. Hruban, D. Hammel, and B. J. Byrne, "Uniform long-term gene expression using adeno-associated virus (AAV) by ex vivo recirculation in rat-cardiac isografts," Thoracic and Cardiovascular Surgeon, vol. 50, no. 6, pp. 347-350, 2002.

[42] Z. Chen, L. Lu, J. Li, X. Xiao, J. J. Fung, and S. Qian, "Prolonged survival of heart allografts transduced with AAVCTLA41g," Microsurgery, vol. 23, no. 5, pp. 489-493, 2003.

[43] A. Doenecke, E. Frank, M. N. Scherer, H. J. Schlitt, and E. K. Geissler, "Prolongation of heart allograft survival after long-term expression of soluble MHC class I antigens and vIL-10 in the liver by AAV-plasmid-mediated gene transfer," Langenbeck's Archives of Surgery, vol. 393, no. 3, pp. 343-348, 2008.

[44] J. M. Schirmer, N. Miyagi, V. P. Rao et al., "Recombinant adeno-associated virus vector for gene transfer to the transplanted rat heart," Transplant International, vol. 20, no. 6, pp. 550-557, 2007.

[45] T. Y. Tsui, X. Wu, C. K. Lau et al., "Prevention of chronic deterioration of heart allograft by recombinant adeno-associated virus-mediated heme oxygenase-1 gene transfer," Circulation, vol. 107, no. 20, pp. 2623-2629, 2003.

[46] P. Gregorevic, M. J. Blankinship, J. M. Allen et al., "Systemic delivery of genes to striated muscles using adeno-associated viral vectors," Nature Medicine, vol. 10, no. 8, pp. 828-834, 2004.

[47] S. J. McNally, J. A. Ross, O. James Garden, and S. J. Wigmore, "Optimization of the paired enzyme assay for heme oxygenase activity," Analytical Biochemistry, vol. 332, no. 2, pp. 398-400, 2004.

[48] R. J. Corry, H. J. Winn, and P. S. Russell, "Primarily vascularized allografts of hearts in mice. The role of $\mathrm{H} 2 \mathrm{D}, \mathrm{H} 2 \mathrm{~K}$, and non $\mathrm{H} 2$ antigens in rejection," Transplantation, vol. 16, no. 4, pp. 343-350, 1973.

[49] K. Sato, J. Balla, L. Otterbein et al., "Carbon monoxide generated by heme oxygenase- 1 suppresses the rejection of mouseto-rat cardiac transplants," Journal of Immunology, vol. 166, no. 6, pp. 4185-4194, 2001.

[50] R. de Waal Malefyt, J. Haanen, H. Spits et al., "Interleukin 10 (IL-10) and viral IL-10 strongly reduce antigen-specific human $\mathrm{T}$ cell proliferation by diminishing the antigenpresenting capacity of monocytes via downregulation of class 
II major histocompatibility complex expression," Journal of Experimental Medicine, vol. 174, no. 4, pp. 915-924, 1991.

[51] J. C. Magee, L. A. DeBruyne, R. Buelow, and J. S. Bromberg, "Gene transfer of immunosuppressive peptides B2702 and RDP1257 prolongs allograft survival: evidence suggesting a role for heme oxygenase-I," Transplantation Proceedings, vol. 31, no. 1-2, p. 1194, 1999.

[52] B. Ke, X. D. Shen, Y. Zhai et al., "Heme oxygenase 1 mediates the immunomodulatory and antiapoptotic effects of interleukin 13 gene therapy in vivo and in vitro," Human Gene Therapy, vol. 13, no. 15, pp. 1845-1857, 2002.

[53] C. Guillot, P. Mathieu, H. Coathalem et al., "Tolerance to cardiac allografts via local and systemic mechanisms after adenovirus-mediated CTLA4Ig expression," Journal of Immunology, vol. 164, no. 10, pp. 5258-5268, 2000.

[54] J. Lee, H. Laks, D. C. Drinkwater et al., "Cardiac gene transfer by intracoronary infusion of adenovirus vector- mediated reporter gene in the transplanted mouse heart," Journal of Thoracic and Cardiovascular Surgery, vol. 111, no. 1, pp. 246252, 1996.

[55] A. H. Schulick, G. Vassalli, P. F. Dunn et al., "Established immunity precludes adenovirus-mediated gene transfer in rat carotid arteries: potential for immunosuppression and vector engineering to overcome barriers of immunity," Journal of Clinical Investigation, vol. 99, no. 2, pp. 209-219, 1997.

[56] C. Zincarelli, S. Soltys, G. Rengo, W. J. Koch, and J. E. Rabinowitz, "Comparative cardiac gene delivery of adenoassociated virus serotypes 1-9 reveals that AAV6 mediates the most efficient transduction in mouse heart," Clinical and Translational Science, vol. 3, no. 3, pp. 81-89, 2010.

[57] L. T. Bish, K. Morine, M. M. Sleeper et al., "Adeno-associated virus (AAV) serotype 9 provides global cardiac gene transfer superior to AAV1, AAV6, AAV7, and AAV8 in the mouse and rat," Human Gene Therapy, vol. 19, no. 12, pp. 1359-1368, 2008.

[58] B. Afzali, G. Lombardi, R. I. Lechler, and G. M. Lord, "The role of T helper 17 (Th17) and regulatory T cells (Treg) in human organ transplantation and autoimmune disease," Clinical and Experimental Immunology, vol. 148, no. 1, pp. 32-46, 2007.

[59] Y. Yamaguchi, K. Fujio, H. Shoda et al., "IL-17B and IL-17C are associated with TNF- $\alpha$ production and contribute to the exacerbation of inflammatory arthritis," Journal of Immunology, vol. 179, no. 10, pp. 7128-7136, 2007.

[60] S. Itoh, N. Kimura, R. C. Axtell et al., "Interleukin-17 accelerates allograft rejection by suppressing regulatory $\mathrm{T}$ cell expansion," Circulation, vol. 124, no. 11, supplement, pp. S187S196, 2011.

[61] A. Awasthi, G. Murugaiyan, and V. K. Kuchroo, "Interplay between effector Th17 and regulatory T cells," Journal of Clinical Immunology, vol. 28, no. 6, pp. 660-670, 2008. 


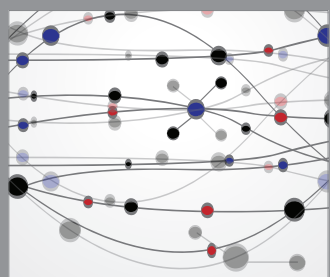

The Scientific World Journal
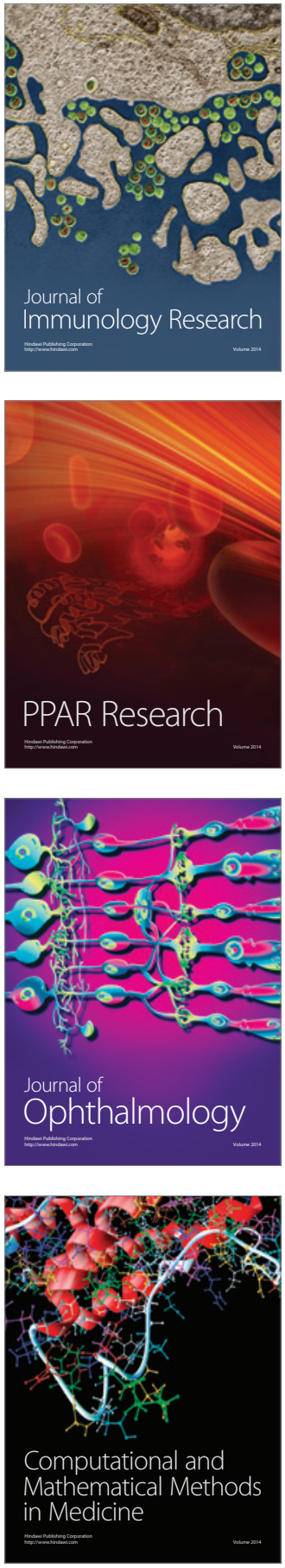

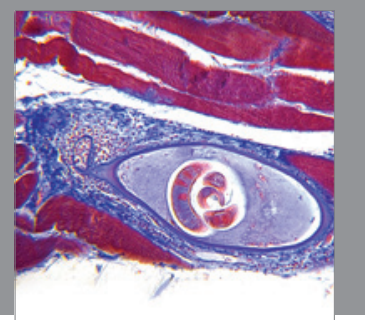

Gastroenterology

Research and Practice
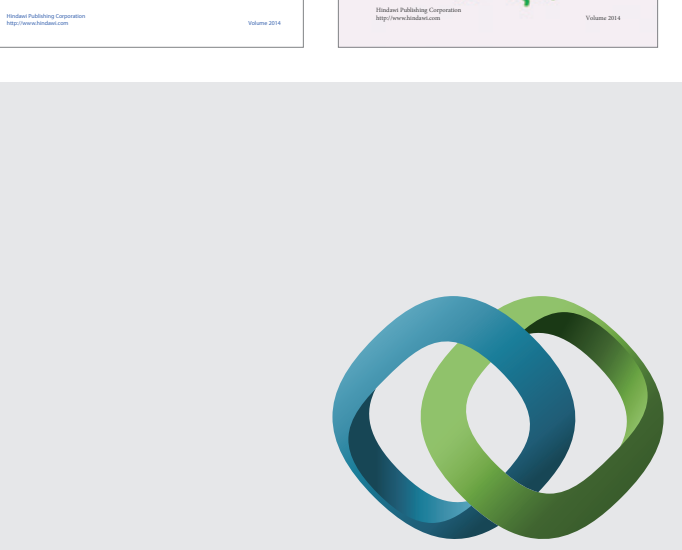

\section{Hindawi}

Submit your manuscripts at

http://www.hindawi.com
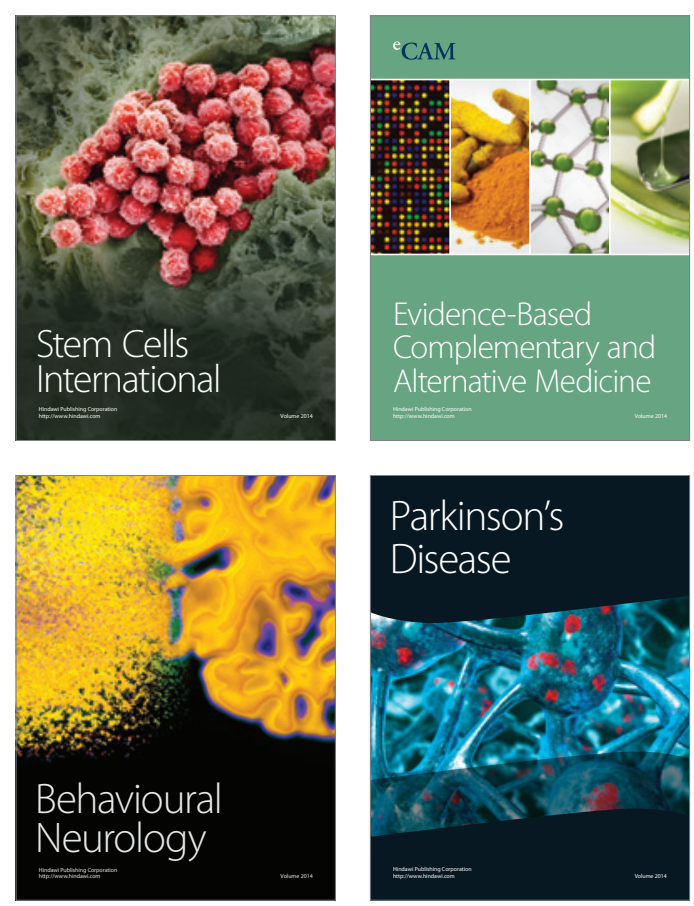

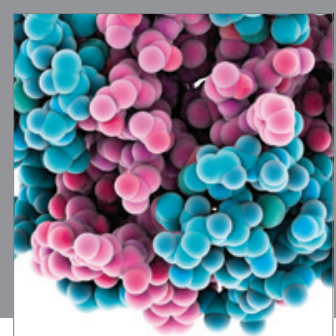

Journal of
Diabetes Research

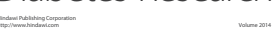

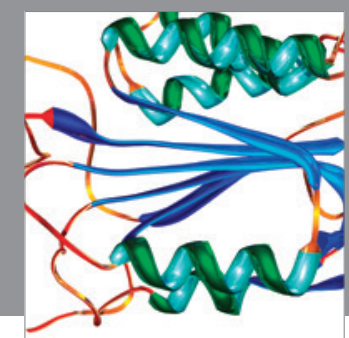

Disease Markers
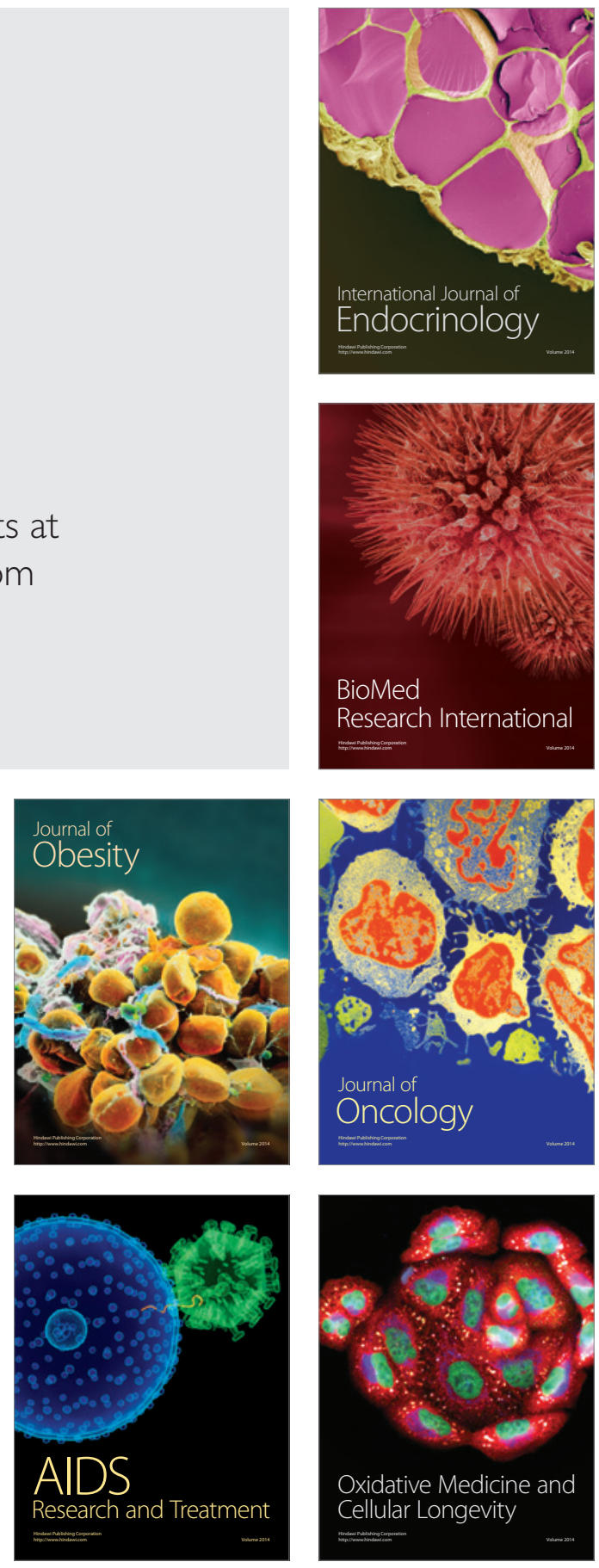